\title{
John-Nirenberg Inequalities with Variable Exponents on Probability Spaces
}

\author{
Lian WU, Zhiwei HAO and Yong JIAO
}

Central South University

(Communicated by H. Kikuchi)

Abstract. In this paper we study the John-Nirenberg inequalities with variable exponents on a probability space. Let $Y$ be a rearrangement invariant Banach function space defined on $(\Omega, \mathcal{F}, P)$ and a measurable function $p(\cdot): \Omega \rightarrow \mathbf{R}^{+}$be a variable exponent. We prove that if the stochastic basis is regular, then

$$
B M O_{\phi, Y}=B M O_{\phi, p(\cdot)}, \quad \forall 1 \leq p(\cdot)<\infty,
$$

where $\phi(r)=1 / r \Phi^{-1}(1 / r)$ and $\Phi$ is a concave function with proper condition.

\section{Introduction}

Let $(\Omega, \mathcal{F}, P)$ be a probability space and $\left\{\mathcal{F}_{n}\right\}_{n \geq 1}$ be a non-decreasing sequence of sub$\sigma$-algebras of $\mathcal{F}$ such that $\mathcal{F}=\sigma\left(\bigcup_{n \geq 1} \mathcal{F}_{n}\right)$. The expectation operator and the conditioned expectation operator are denoted by $\mathbf{E}$ and $\mathbf{E}_{n}$, resp. A sequence $f=\left(f_{n}\right)_{n \geq 1}$ of random variables such that $f_{n}$ is $\mathcal{F}_{n}$-measurable is said to be a martingale if $\mathbf{E}\left(\left|f_{n}\right|\right)<\infty$ and $\mathbf{E}_{n}\left(f_{n+1}\right)=f_{n}$ for every $n \geq 1$. For $1 \leq p<\infty$, the Banach space $B M O_{p}$ is defined as:

$$
B M O_{p}=\left\{f=\left(f_{n}\right)_{n \geq 1}:\|f\|_{B M O_{p}}=\sup _{n}\left\|\mathbf{E}_{n}\left(\left|f-f_{n}\right|^{p}\right)\right\|_{\infty}^{\frac{1}{p}}<\infty\right\},
$$

where the $f$ in $\left|f-f_{n}\right|^{p}$ means $f_{\infty}$. Let $\mathcal{T}$ be the set of all stopping times with respect to $\left\{\mathcal{F}_{n}\right\}_{n \geq 1}$. It is easy to check that (see for instance Theorem 4.2 .5 in [14] or [31])

$$
\sup _{n}\left\|\mathbf{E}_{n}\left(\left|f-f_{n}\right|^{p}\right)\right\|_{\infty}^{\frac{1}{p}}=\sup _{v \in \mathcal{T}}(P(v<\infty))^{-\frac{1}{p}}\left\|\left(f-f^{v}\right) \chi_{\{v<\infty\}}\right\|_{p} .
$$

Received March 25, 2014; revised July 23, 2014

2000 Mathematics Subject Classification: 60G42, 60G46

Key words and phrases: John-Nirenberg inequalities, martingales, variable exponents, rearrangement invariant spaces

Lian Wu is partially supported by the Fundamental Research Funds for the Central Universities of Central South University: 2013zzts007.

Yong Jiao is supported by NSFC(11471337), Hunan Provincial Natural Science Foundation(14JJ1004) and The International Postdoctoral Exchange Fellowship Program. 
We also refer to [15] for the Carleson measures characterization of BMO norms. Based mainly on duality, John-Nirenberg's theorem and something else, the space BMO (Bounded Mean Oscillation; see John-Nirenberg [18]) plays an important role in classical analysis and martingale theory. The well known John-Nirenberg Theorem (one of the most important theorems in martingale theory) says that if the stochastic basis $\left\{\mathcal{F}_{n}\right\}_{n \geq 1}$ is regular, then

$$
B M O_{p}=B M O_{1} .
$$

We refer to [11] and [12] for the fact above. Recently, Miyamoto, Nakai and Sadasue [26] extend (1.1) to a much more general setting. We briefly recall their result. Let $\mathfrak{S}$ be the set of all functions $\Phi:[0, \infty) \rightarrow[0, \infty)$ satisfying $\Phi(0)=0, \lim _{r \rightarrow \infty} \Phi(r)=\infty$. Denote by $\mathfrak{S}_{\ell}$ the set

$$
\mathfrak{S}_{\ell}=\left\{\Phi \in \mathfrak{S}: \exists c_{\Phi} \geq 1 \text { and } \ell \in(0,1] \text { s.t. } \Phi(t r) \leq c_{\Phi} \max \left\{t^{\ell}, t\right\} \Phi(r) \text { for } t, r \in[0, \infty)\right\} .
$$

For $q \in[1, \infty)$ and a function $\phi:(0, \infty) \rightarrow(0, \infty)$, the generalized Campanato martingale space $B M O_{\phi, q}$ is defined by

$$
B M O_{\phi, q}=\left\{f=\left(f_{n}\right)_{n \geq 1}:\|f\|_{B M O_{\phi, q}}<\infty\right\},
$$

where

$$
\|f\|_{B M O_{\phi, q}}=\sup _{n \geq 1} \sup _{A \in \mathcal{F}_{n}} \frac{1}{\phi(P(A))}\left(\frac{1}{P(A)} \int_{A}\left|f-\mathbf{E}_{n} f\right|^{q} d P\right)^{1 / q} .
$$

Then if there exists $\Phi \in \mathfrak{S}_{\ell}$ such that $\phi(r)=\frac{1}{r \Phi^{-1}(1 / r)}$ and the stochastic basis is regular, the John-Nirenberg type inequality holds. Namely, for all $1 \leq q<\infty$

$$
B M O_{\phi, q}=B M O_{\phi, 1} \text {. }
$$

On the other hand, we also note that in the past few years the subject of variable exponent spaces has undergone a vast development. We refer to two monographs $[3,6]$ for the recent progress on variable Lebesgue spaces and their applications to partial differential equations and variational integrals with non-standard growth conditions, and [5, 29] for the variable Hardy spaces.

Although variable exponent Lebesgue spaces on Euclidean space have attracted a steadily increasing interest, the variable exponent framework has not yet been applied to the probability space setting. Compared with Euclidean spaces, the essential difficulty is that the general probability space has no natural metric structure to define the so-called log-Hölder continuity of variable exponents. In the present paper, we find the following condition without metric characterization of $p(x)$ to replace log-Hölder continuity in some sense. That is, there exists an absolute constant $K_{p(\cdot)} \geq 1$ depending only on $p(\cdot)$ such that

$$
\mathbf{P}(A)^{p_{-}(A)-p_{+}(A)} \leq K_{p(\cdot)}, \quad \forall A \in \mathcal{F} .
$$


See Section 2 for the notations. We often denote $K_{p(\cdot)}$ simply by $K$ if there is nothing confused. Under the condition of (1.3), we study the John-Nirenberg inequalities with variable exponents on a probability space. We refer to the very recent paper [17] for the Doob maximal inequality and atomic decomposition on variable Hardy martingale spaces.

Now let us briefly state the main result in the present paper. Let $Y$ be a rearrangement invariant Banach function space and $p(\cdot): \Omega \rightarrow \mathbf{R}^{+}$be a variable exponent. We define the generalized Campanato martingale spaces associated with $Y$

$$
B M O_{\phi, Y}=\left\{f=\left(f_{n}\right)_{n \geq 1}:\|f\|_{B M O_{\phi, Y}}<\infty\right\},
$$

where

$$
\|f\|_{B M O_{\phi, Y}}=\sup _{v \in \mathcal{T}} \frac{1}{\phi(P(v<\infty))} \frac{\left\|\left(f-f^{v}\right) \chi_{\{v<\infty\}}\right\|_{Y}}{\left\|\chi_{\{v<\infty\}}\right\|_{Y}} ;
$$

and we also define the generalized Campanato martingale space with variable exponent $p(\cdot)$

$$
B M O_{\phi, p(\cdot)}=\left\{f=\left(f_{n}\right)_{n \geq 1}:\|f\|_{B M O_{\phi, p(\cdot)}}<\infty\right\},
$$

where

$$
\|f\|_{B M O_{\phi, p(\cdot)}}=\sup _{v \in \mathcal{T}} \frac{1}{\phi(P(v<\infty))} \frac{\left\|\left(f-f^{v}\right) \chi_{\{v<\infty\}}\right\|_{p(\cdot)}}{\left\|\chi_{\{v<\infty\}}\right\|_{p(\cdot)}} .
$$

Let $\Phi \in \mathfrak{S}_{\ell}$ and $\phi(r)=\frac{1}{r \Phi^{-1}(1 / r)}$. The main result in this paper is if the stochastic basis is regular and $p(\cdot)$ satisfies the condition (1.3), then

$$
B M O_{\phi, Y}=B M O_{\phi, p(\cdot)}, \quad \forall 1 \leq p(\cdot)<\infty .
$$

Our strategy is to prove that $B M O_{\phi, Y}$ and $B M O_{\phi, p(\cdot)}$ are respectively equivalent to $B M O_{\phi, 1}$. The present results can be regarded as probability versions of [20,21, 22]; however the method is very different, and the condition (1.3) makes us avoid the use of log-Hölder continuity or the boundedness of Hardy-Littlewood maximal operator.

Throughout this paper, we always denote by $C$ an absolute positive constant, which can vary from line to line, and denote by $C_{p(\cdot)}$ the constant depending only on $p(\cdot)$. The symbol $A \lesssim B$ stands for the inequality $A \leq C B$ for some constant $C$. If we write $A \approx B$, then it stands for $A \lesssim B \lesssim A$.

\section{Preliminaries}

In this section, we give some preliminaries necessary to the whole paper.

2.1. Rearrangement invariant Banach function spaces. Let us first recall some basic facts on the rearrangement invariant Banach function spaces. Given two random variables $f$ and $g$, we write $f \stackrel{d}{\simeq} g$ to mean that they have the same distribution. A Banach function 
space $\left(Y,\|\cdot\|_{Y}\right)$ is said to be a rearrangement invariant Banach function space if $f \stackrel{d}{\simeq} g$ and $g \in Y$, then $f \in Y$ and $\|f\|_{Y}=\|g\|_{Y}$. We refer to Chapter 2 in [2] for the more concrete definition.

A rearrangement invariant Banach function space is simply called an r.i. space in this paper. For example, Lebesgue spaces, Orlicz spaces and Lorentz spaces are r.i. spaces, while weighted Lebesgue spaces with suitable weights are Banach function spaces that are not ri. spaces.

In order to describe our results we need the Boyd indices of $Y$ introduced by Boyd in [9]. We define, for every $0<c<\infty$, the dilation operator $D_{c}$ acting on the space of measurable functions on $[0,1]$ by $D_{c}(f)(t)=f(c t)$, if $0<c t \leq 1 ; D_{c}(f)(t)=0$, otherwise. The lower Boyd index and the upper Boyd index of $Y$ are respectively defined by

$$
\begin{aligned}
& \alpha_{Y}=\lim _{c \rightarrow 0+} \frac{\log \left\|D_{c^{-1}}\right\|}{\log c}=\sup _{0<c<1} \frac{\log \left\|D_{c^{-1}}\right\|}{\log c}, \\
& \beta_{Y}=\lim _{c \rightarrow \infty} \frac{\log \left\|D_{c^{-1}}\right\|}{\log c}=\inf _{1<c<\infty} \frac{\log \left\|D_{c^{-1}}\right\|}{\log c},
\end{aligned}
$$

where $\left\|D_{c}\right\|$ stands for the operator norm of $D_{c}: Y \longrightarrow Y$. Noting that for any r.i. space $Y$,

$$
0 \leq \alpha_{Y} \leq \beta_{Y} \leq 1 .
$$

We shall need the following fact for Boyd indices (see [24], Theorem II.4.110). If $Y$ is an r.i. space on $\Omega$ with associate space $Y^{\prime}$, then

$$
\alpha_{Y}+\beta_{Y^{\prime}}=1, \quad \alpha_{Y^{\prime}}+\beta_{Y}=1 .
$$

The following lemmas can be found in [2].

LEMMA 2.1. Let $Y$ be an ri. space on $\Omega$ with associate space $Y^{\prime}$. If $f \in Y$ and $g \in Y^{\prime}$, then $f g$ is integrable and

$$
\int_{\Omega}|f(\omega) g(\omega)| d P \leq\|f\|_{Y}\|g\|_{Y^{\prime}} .
$$

LEMMA 2.2. Let $Y$ be an ri. space on $\Omega$ with associate space $Y^{\prime}$. Then

$$
\left\|\chi_{A}\right\|_{Y}\left\|\chi_{A}\right\|_{Y^{\prime}}=P(A),
$$

for any measurable set $A \subset \Omega$ with $P(A)<\infty$.

LEMMA 2.3. Let $Y$ be an ri. space on $\Omega$ with associate space $Y^{\prime}$. Then

$$
\|f\|_{Y}=\sup _{\|h\|_{Y^{\prime}} \leq 1}\left|\int_{\Omega} f(\omega) h(\omega) d P\right| .
$$


2.2. Variable exponents. In this subsection, we introduce variable exponent Lebesgue spaces $L^{p(\cdot)}(\Omega)$ defined on a probability space $(\Omega, \mathcal{F}, P)$ and some useful lemmas.

DEFINITION 2.4. Given a probability space $(\Omega, \mathcal{F}, P)$, a measurable functions $p(\cdot)$ : $\Omega \longrightarrow[1, \infty)$ is called a variable exponent. Let $\mathcal{P}(\Omega)$ be the set of all variable exponents.

Let $A \subset \Omega$. We denote

$$
p_{+}(A)=\sup _{x \in A} p(x), \quad p_{-}(A)=\inf _{x \in A} p(x) .
$$

If $A=\Omega$, we simply write $p_{+}=p_{+}(\Omega)$ and $p_{-}=p_{-}(\Omega)$. Given $p(\cdot) \in \mathcal{P}$, we always assume that $p_{+}<\infty$. The space $L^{p(\cdot)}=L^{p(\cdot)}(\Omega)$ is the collection of all measurable functions $f$ such that for some $\lambda>0$,

$$
\rho(f / \lambda)=\int_{\Omega}\left(\frac{|f(x)|}{\lambda}\right)^{p(x)} d P<\infty .
$$

This becomes a Banach function space when equipped with the Luxemburg norm

$$
\|f\|_{p(\cdot)} \equiv \inf \{\lambda>0: \rho(f / \lambda) \leq 1\} .
$$

We note that $L^{p(\cdot)}$ is not an r.i. space generally.

Lemma 2.5 (see [3], page 24). Given $p(\cdot) \in \mathcal{P}$, then for all $f \in L^{p(\cdot)}$ and $\|f\|_{p(\cdot)} \neq$ 0 , we have

$$
\int_{\Omega}\left|\frac{f}{\|f\|_{p(\cdot)}}\right|^{p(x)} d P=1 .
$$

Lemma 2.6 (see [10], Theorem 1.3). Given $p(\cdot) \in \mathcal{P}$ and $f \in L^{p(\cdot)}$, then we have

(1) $\|f\|_{p(\cdot)}<1(=1,>1)$ if and only if $\rho(f)<1(=1,>1)$;

(2) If $\|f\|_{p(\cdot)}>1$, then $\rho(f)^{1 / p_{+}} \leq\|f\|_{p(\cdot)} \leq \rho(f)^{1 / p_{-}}$;

(3) If $0<\|f\|_{p(\cdot)} \leq 1$, then $\rho(f)^{1 / p_{-}} \leq\|f\|_{p(\cdot)} \leq \rho(f)^{1 / p_{+}}$.

LEMma 2.7 (see [3], Hölder's inequality). Given $p(\cdot), q(\cdot), r(\cdot) \in \mathcal{P}$, such that

$$
\frac{1}{p(x)}=\frac{1}{q(x)}+\frac{1}{r(x)} .
$$

Then there exists a constant $C_{p(\cdot)}$ such that for all $f \in L^{q(\cdot)}$ and $g \in L^{r(\cdot)}, f g \in L^{p(\cdot)}$ and

$$
\|f g\|_{p(\cdot)} \leq C_{p(\cdot)}\|f\|_{q(\cdot)}\|g\|_{r(\cdot)} .
$$

Definition 2.8. Given $p(\cdot) \in \mathcal{P}$ and a function $\phi:(0, \infty) \rightarrow(0, \infty)$, the generalized Campanato martingale space with variable exponent $B M O_{\phi, p(\cdot)}$ is defined by

$$
B M O_{\phi, p(\cdot)}=\left\{f=\left(f_{n}\right)_{n \geq 1}:\|f\|_{B M O_{\phi, p(\cdot)}}<\infty\right\}
$$


where

$$
\|f\|_{B M O_{\phi, p(\cdot)}}=\sup _{v \in \mathcal{T}} \frac{1}{\phi(P(v<\infty))} \frac{\left\|\left(f-f^{v}\right) \chi_{\{v<\infty\}}\right\|_{p(\cdot)}}{\left\|\chi_{\{v<\infty\}}\right\|_{p(\cdot)}} .
$$

2.3. Hardy-Orlicz martingale spaces. Let $\Phi \in \mathfrak{S}_{\ell}$ with $\ell \in(0,1]$. The Orlicz space $L_{\Phi}$ is defined as all measurable functions $f$ with respect to $(\Omega, \mathcal{F}, P)$ such that $\mathbf{E}(\Phi(c|f|))<\infty$ for some $c>0$ and

$$
\|f\|_{L_{\Phi}}=\inf \{c>0: \mathbf{E}(\Phi(|f| / c)) \leq 1\} .
$$

Now we define the Orlicz-Hardy martingale spaces. For a martingale $f=\left(f_{n}\right)_{n \geq 1}$, we denote its martingale difference by $d f_{i}=f_{i}-f_{i-1}$ (with convention $f_{0}=0$ ). Then the conditional quadratic variation, the square function and the maximal function of martingale $f$ are defined by

$$
\begin{array}{cl}
s_{n}(f)=\left(\sum_{i=1}^{n} \mathbf{E}_{i-1}\left|d f_{i}\right|^{2}\right)^{1 / 2}, & s(f)=\left(\sum_{i=1}^{\infty} \mathbf{E}_{i-1}\left|d f_{i}\right|^{2}\right)^{1 / 2} ; \\
S_{n}(f)=\left(\sum_{i=1}^{n}\left|d f_{i}\right|^{2}\right)^{1 / 2}, & S(f)=\left(\sum_{i=1}^{\infty}\left|d f_{i}\right|^{2}\right)^{1 / 2} ; \\
f_{n}^{*}=\sup _{1 \leq i \leq n}\left|f_{i}\right|, \quad f^{*}=\sup _{i \geq 1}\left|f_{i}\right| .
\end{array}
$$

For $\Phi \in \mathfrak{S}_{\ell}$, we define

$$
\begin{aligned}
& H_{\Phi}^{S}=\left\{f=\left(f_{n}\right)_{n \geq 1}:\|f\|_{H_{\Phi}^{s}}=\|s(f)\|_{L_{\Phi}}<\infty\right\} . \\
& H_{\Phi}^{S}=\left\{f=\left(f_{n}\right)_{n \geq 1}:\|f\|_{H_{\Phi}^{S}}=\|S(f)\|_{L_{\Phi}}<\infty\right\} ; \\
& H_{\Phi}=\left\{f=\left(f_{n}\right)_{n \geq 1}:\|f\|_{H_{\Phi}}=\left\|f^{*}\right\|_{L_{\Phi}}<\infty\right\} .
\end{aligned}
$$

The stochastic basis $\left(\mathcal{F}_{n}\right)_{n \geq 1}$ is said to be regular, if for $n \geq 0$ and $A \in \mathcal{F}_{n}$, there exists $B \in \mathcal{F}_{n-1}$ such that $A \subset B$ and $P(B) \leq R P(A)$, where $R$ is a positive constant independent of $n$. A martingale is said to be regular if it is adapted to a regular $\sigma$-algebra sequence. This amounts to saying that there exists a constant $R>0$ such that

$$
f_{n} \leq R f_{n-1}
$$

for all nonnegative martingales $\left(f_{n}\right)_{n \geq 1}$ adapted to the stochastic basis $\left(\mathcal{F}_{n}\right)_{n \geq 1}$. For $\Phi \in \mathfrak{S}_{\ell}$, the authors in [26] proved that $H_{\Phi}=H_{\Phi}^{S}=H_{\Phi}^{S}$ when the stochastic basis $\left(\mathcal{F}_{n}\right)_{n \geq 1}$ is regular, and that the dual space of $H_{\Phi}^{s}$ is $B M O_{\phi, 2}$, where $\phi(r)=\frac{1}{r \Phi^{-1}(1 / r)}$. We end this section by collecting two useful properties of $\Phi \in \mathfrak{S}_{\ell}$. For the detailed proof of them, we refer to [26]. 
Proposition 2.9. If $\Phi \in \mathfrak{S}_{\ell}$ with $\ell \in(0,1]$, then

(1) $\Phi$ is equivalent to a concave function in $\mathfrak{S}_{\ell}$;

(2) $\Phi$ is subadditive.

\section{Main results}

THEOREM 3.1. Let $Y$ be an ri. Banach function space with $\alpha_{Y}>0$ and $p(\cdot) \in \mathcal{P}$. If there exists $\Phi \in \mathfrak{S}_{\ell}$ with $\ell \in(0,1]$ such that $\phi(r)=\frac{1}{r \Phi^{-1}(1 / r)}$ for all $r \in(0, \infty)$, and $\left(\mathcal{F}_{n}\right)_{n \geq 1}$ is regular, then

$$
B M O_{\phi, Y}=B M O_{\phi, p(\cdot)} .
$$

3.1. John-Nirenberg inequalities with r.i. spaces. In this subsection we prove $B M O_{\phi, Y}=B M O_{\phi, 1}$ by constructing atomic decomposition of Orlicz-Hardy spaces $H_{\Phi}^{s}$ via atoms associated with r.i. spaces.

Definition 3.2. Let $\Phi \in \mathfrak{S}_{\ell}$ with $\ell \in(0,1]$ and $Y$ be an r.i. space on $\Omega$ with $\beta_{Y}<1$. A measurable function $a$ is a $(\Phi, Y)^{S}$-atom $\left(\right.$ or $(\Phi, Y)^{S},(\Phi, Y)^{*}$ resp.) if there exists a stopping time $v \in \mathcal{T}$ such that

(a1) $a_{n}=\mathbf{E}_{n} a=0$ if $v \geq n$;

(a2) $\|s(a)\|_{Y} \leq \frac{\left\|\chi_{\{v<\infty\}}\right\|_{Y}}{\left\|\chi_{\{v<\infty\}}\right\|_{L_{\Phi}}}$ (or $\|S(a)\|_{Y},\left\|a^{*}\right\|_{Y}$ resp.).

Denote by $A^{s}(\Phi, Y)$ (or $\left.A^{*}(\Phi, Y)\right)$ be the set of all sequences of pair $\left(\mu_{k}, a^{k}, v_{k}\right)$, where $\mu_{k}$ are nonnegative numbers, $a^{k}$ are $(\Phi, Y)^{s}$-atoms (or $(\Phi, Y)^{*}$-atoms) and $v_{k}$ are the stopping times corresponding to $a^{k}$ and also

$$
\begin{gathered}
\sum_{k \in \mathbf{Z}} P\left(v_{k}<\infty\right) \Phi\left(\frac{\mu_{k}\left\|s\left(a^{k}\right)\right\|_{Y}}{\left\|\chi_{\left\{v_{k}<\infty\right\}}\right\|_{Y}}\right)<\infty \\
\left(\text { or } \sum_{k \in \mathbf{Z}} P\left(v_{k}<\infty\right) \Phi\left(\frac{\mu_{k}\left\|\left(a^{k}\right)^{*}\right\|_{Y}}{\left\|\chi_{\left\{v_{k}<\infty\right\}}\right\|_{Y}}\right)<\infty .\right)
\end{gathered}
$$

Let $\left(\mu_{k}, a^{k}, v_{k}\right) \in A^{s}(\Phi, Y)$ (or $\left.A^{*}(\Phi, Y)\right)$ and define

$$
\begin{gathered}
\mathcal{A}_{Y}^{s}\left(\mu_{k}, a^{k}, v_{k}\right)=\inf \left\{c>0: \sum_{k \in \mathbf{Z}} P\left(v_{k}<\infty\right) \Phi\left(\frac{\mu_{k}\left\|s\left(a^{k}\right)\right\|_{Y}}{c\left\|\chi_{\left\{v_{k}<\infty\right\}}\right\|_{Y}}\right) \leq 1\right\} \\
\left(\operatorname{or} \mathcal{A}_{Y}^{*}\left(\mu_{k}, a^{k}, v_{k}\right)=\inf \left\{c>0: \sum_{k \in \mathbf{Z}} P\left(v_{k}<\infty\right) \Phi\left(\frac{\mu_{k}\left\|\left(a^{k}\right)^{*}\right\|_{Y}}{c\left\|\chi_{\left\{v_{k}<\infty\right\}}\right\|_{Y}}\right) \leq 1\right\} .\right)
\end{gathered}
$$

In order to prove the main theorem, we need the following lemma. 
LEMMA 3.3. Let $\Phi \in \mathfrak{S}_{\ell}$ with $\ell \in(0,1]$ and $Y$ be an ri. Banach function space. For $f \in Y$ and $B \in \mathcal{F}$ with $P(B) \neq 0$, if $\{f \neq 0\} \subset B$, then

$$
\mathbf{E}(\Phi(|f|)) \leq 2 P(B) \Phi\left(\frac{\|f\|_{Y}}{\left\|\chi_{B}\right\|_{Y}}\right) .
$$

PROOF. Set $m=\frac{\|f\|_{Y}}{\left\|\chi_{B}\right\|_{Y}}$. Since $t \Phi(r) \leq \Phi(t r)$ holds for $t \in[0,1]$ and $r \in[0, \infty)$, we have

$$
\Phi(|f|)=\Phi\left(|f| \chi_{B}\right) \leq \Phi(m+|f|) \chi_{B} \leq\left(1+\frac{|f|}{m}\right) \Phi(m) \chi_{B} .
$$

Thus, by Lemma 2.1 and Lemma 2.2 we obtain the conclusion as follows:

$$
\begin{aligned}
\mathbf{E}(\Phi(|f|)) & \leq \mathbf{E}\left(\left(1+\frac{|f|}{m}\right) \Phi(m) \chi_{B}\right) \\
& =\Phi(m) \mathbf{E}\left(\left(1+\frac{|f|}{m}\right) \chi_{B}\right) \\
& =\Phi(m)\left(\mathbf{E}\left(\chi_{B}\right)+\mathbf{E}\left(\frac{|f|}{m} \chi_{B}\right)\right) \\
& \leq \Phi(m)\left(P(B)+\left\|\frac{|f|}{m}\right\|_{Y}\left\|\chi_{B}\right\|_{Y^{\prime}}\right) \\
& =\Phi(m)\left(P(B)+\left\|\chi_{B}\right\|_{Y}\left\|_{B}\right\|_{Y^{\prime}}\right) \\
& =2 \Phi(m) P(B) .
\end{aligned}
$$

REMARK 3.4. By Lemma 3.3, we have that, for any positive constant $\mu$ and for any pair $(a, v)$ of $(\Phi, Y)^{s}$-atom,

$$
\begin{aligned}
\int_{\Omega} \Phi\left(\frac{\mu s(a)}{\left(2 c_{\Phi}\right)^{1 / \ell}}\right) d P & \leq \frac{1}{2} \int_{\Omega} \Phi(\mu s(a)) d P \\
& \leq P(v<\infty) \Phi\left(\frac{\mu\|s(a)\|_{Y}}{\left\|\chi_{\{v<\infty\}}\right\|_{Y}}\right) .
\end{aligned}
$$

THEOREM 3.5. Let $\Phi \in \mathfrak{S}_{\ell}$ with $\ell \in(0,1]$ and $Y$ be an r.i. Banach function space with $\beta_{Y}<1$. Then

$$
\|f\|_{H_{\Phi}^{s}} \approx \inf \left\{\mathcal{A}_{Y}^{s}\left(\mu_{k}, a^{k}, v_{k}\right): f=\sum_{k \in \boldsymbol{Z}} \mu_{k} a^{k}, \quad\left(\mu_{k}, a^{k}, v_{k}\right) \in A^{s}(\Phi, Y)\right\} .
$$

Proof. Suppose that $f \in H_{\Phi}^{s}$. Then from [26] Theorem 2.3, there exists a sequence of pair $\left(\mu_{k}, a^{k}, v_{k}\right) \in A^{s}\left(\Phi, L_{\infty}\right)$ such that $f=\sum_{k \in \mathbf{Z}} \mu_{k} a^{k}$ and $\mathcal{A}_{L_{\infty}}^{s}\left(\mu_{k}, a^{k}, v_{k}\right) \leq c\|f\|_{H_{\Phi}^{s}}$, where $c$ is a constant independent of $f$. By the proof of [26] Theorem 2.3, we know that $\mathbf{E}_{n} a^{k}=0$ if $v_{k} \geq n$ and

$$
s\left(a^{k}\right)=0 \text { on the set }\left\{v_{k}=\infty\right\}
$$


where $v_{k}$ is the stopping time corresponding to $a^{k}$. Thus

$$
s\left(a^{k}\right) \leq\left\|s\left(a^{k}\right)\right\|_{\infty} \chi_{\left\{v_{k}<\infty\right\}} .
$$

Applying $\|\cdot\|_{Y}$ on both sides of the above inequality, we find that

$$
\begin{aligned}
\left\|s\left(a^{k}\right)\right\|_{Y} & \leq\left\|s\left(a^{k}\right)\right\|_{\infty}\left\|\chi_{\left\{v_{k}<\infty\right\}}\right\|_{Y} \\
& \leq \frac{\left\|\chi_{\left\{v_{k}<\infty\right\}}\right\|_{Y}}{\| \chi\left\{v_{k}<\infty\right\}},
\end{aligned}
$$

which means (a2) holds. And so for each $k, a^{k}$ is a $(\Phi, Y)^{s}$-atom.

Next we show that $\left(\mu_{k}, a^{k}, v_{k}\right) \in A^{s}(\Phi, Y)$. Since

$$
\begin{aligned}
& \sum_{k \in \mathbf{Z}} P\left(v_{k}<\infty\right) \Phi\left(\frac{\mu_{k}\left\|s\left(a^{k}\right)\right\|_{Y}}{\mathcal{A}_{L_{\infty}}^{s}\left(\mu_{k}, a^{k}, v_{k}\right)\left\|\chi_{\left\{v_{k}<\infty\right\}}\right\|_{Y}}\right) \\
\leq & \sum_{k \in \mathbf{Z}} P\left(v_{k}<\infty\right) \Phi\left(\frac{\mu_{k}\left\|s\left(a^{k}\right)\right\|_{\infty}\left\|\chi_{\left\{v_{k}<\infty\right\}}\right\|_{Y}}{\mathcal{A}_{L_{\infty}}^{s}\left(\mu_{k}, a^{k}, v_{k}\right)\left\|\chi_{\left\{v_{k}<\infty\right\}}\right\|_{Y}}\right) \\
= & \sum_{k \in \mathbf{Z}} P\left(v_{k}<\infty\right) \Phi\left(\frac{\mu_{k}\left\|s\left(a^{k}\right)\right\|_{\infty}}{\mathcal{A}_{L_{\infty}}^{s}\left(\mu_{k}, a^{k}, v_{k}\right)}\right) \\
\leq & 1,
\end{aligned}
$$

which means

$$
\mathcal{A}_{Y}^{s}\left(\mu_{k}, a^{k}, v_{k}\right) \leq \mathcal{A}_{L_{\infty}}^{s}\left(\mu_{k}, a^{k}, v_{k}\right) \leq c\|f\|_{H_{\Phi}^{s}},
$$

namely, $\left(\mu_{k}, a^{k}, v_{k}\right) \in A^{s}(\Phi, Y)$.

Moreover, again from the proof of [26] Theorem 2.3, $\sum_{k=m}^{n} \mu_{k} a^{k}$ converges to $f$ in $H_{\Phi}^{s}$ as $m \rightarrow-\infty, n \rightarrow \infty$.

For the converse part, assume that $f=\sum_{k \in \mathbf{Z}} \mu_{k} a^{k}$ and $\left(\mu_{k}, a^{k}, v_{k}\right) \in A^{s}(\Phi, Y)$. And without loss of generality, we suppose that $\mathcal{A}_{Y}^{s}\left(\mu_{k}, a^{k}, v_{k}\right)=1$. Then by the subadditivity of $\Phi$ and Remark 3.4, we have

$$
\begin{aligned}
\int_{\Omega} \Phi\left(\frac{s(f)}{\left(2 c_{\Phi}\right)^{1 / \ell}}\right) d P & \leq \int_{\Omega} \Phi\left(\frac{\sum_{k \in \mathbf{Z}} \mu_{k} s\left(a^{k}\right)}{\left(2 c_{\Phi}\right)^{1 / \ell}}\right) d P \\
& \leq \sum_{k \in \mathbf{Z}} \int_{\Omega} \Phi\left(\frac{\mu_{k} s\left(a^{k}\right)}{\left(2 c_{\Phi}\right)^{1 / \ell}}\right) d P \\
& \leq \sum_{k \in \mathbf{Z}} P\left(v_{k}<\infty\right) \Phi\left(\frac{\mu_{k}\left\|s\left(a^{k}\right)\right\|_{Y}}{\left\|\chi_{\left\{v_{k}<\infty\right\}}\right\|_{Y}}\right) \\
& \leq 1 .
\end{aligned}
$$

This shows $\|f\|_{H_{\Phi}^{s}} \leq\left(2 c_{\Phi}\right)^{1 / \ell} \mathcal{A}_{Y}^{s}\left(\mu_{k}, a^{k}, v_{k}\right)$. The proof is complete. 
REMARK 3.6. 1) The condition $\beta_{Y}<1$ can be replaced by a weaker condition which could be described by Orlicz indices. It is a well known fact that one has no atomic decomposition for $\Phi(t)=t$ in classical harmonic analysis when $Y=L_{1}$ (see Theorem 4.10 in [4] ), and that's why we have such a condition.

2) If $\left(\mathcal{F}_{n}\right)_{n \geq 1}$ is regular, then $H_{\Phi}=H_{\Phi}^{s}=H_{\Phi}^{S}$ for $\Phi \in \mathfrak{S}_{\ell}$, see [26]. Hence we can also obtain the $(\Phi, Y)^{S}$ (or $(\Phi, Y)^{*}$ resp.)-atomic decomposition for $H_{\Phi}^{S}$ (or $H_{\Phi}$ resp.). The proof is similar to the one of Theorem 3.5, so we leave the details to the reader.

THEOREM 3.7. Let $Y$ be an ri. Banach function space with $\alpha_{Y}>0$. If there exists $\Phi \in \mathfrak{S}_{\ell}$ with $\ell \in(0,1]$ such that $\phi(r)=\frac{1}{r \Phi^{-1}(1 / r)}$ for all $r \in(0, \infty)$, and $\left(\mathcal{F}_{n}\right)_{n \geq 1}$ is regular, then

$$
B M O_{\phi, Y}=B M O_{\phi, 1} .
$$

Proof. First suppose that $f \in B M O_{\phi, Y}$. Then for $v \in \mathcal{T}$, by Lemmas 2.1 and 2.2, we have

$$
\begin{aligned}
& \frac{1}{\phi(P(v<\infty))} \frac{\left\|\left(f-f^{v}\right) \chi_{\{v<\infty\}}\right\|_{1}}{P(v<\infty)} \\
\leq & \frac{1}{\phi(P(v<\infty))} \frac{\left\|\left(f-f^{v}\right) \chi_{\{v<\infty\}}\right\|_{Y}\left\|_{\{v<\infty\}}\right\|_{Y^{\prime}}}{P(v<\infty)} \\
= & \frac{1}{\phi(P(v<\infty))} \frac{\left\|\left(f-f^{v}\right) \chi_{\{v<\infty\}}\right\|_{Y}}{\left\|\chi_{\{v<\infty\}}\right\|_{Y}} .
\end{aligned}
$$

This means $\|f\|_{B M O_{\phi, 1}} \leq\|f\|_{B M O_{\phi, Y}}$.

On the other hand, assume that $f \in B M O_{\phi, 1}$. Then for $v \in \mathcal{T}$, by Lemma 2.3, there exists $h \in Y^{\prime}$ and $\|h\|_{Y^{\prime}} \leq 1$ such that

$$
\left\|\left(f-f^{v}\right) \chi_{\{v<\infty\}}\right\|_{Y} \leq 2\left|\int_{\{v<\infty\}}\left(f-f^{v}\right) h d P\right| .
$$

By the Doob inequality in r.i. spaces, let $c_{0}$ be the constant (depending only on $Y^{\prime}$ ) such that $\left\|f^{*}\right\|_{Y^{\prime}} \leq c_{0}\|f\|_{Y^{\prime}}, \forall f \in Y^{\prime}$; see for instance [28]. Define

$$
a=\frac{\|\chi\{v<\infty\}\|_{Y^{\prime}}\left(h-h^{v}\right)}{2 c_{0}\|\chi\{v<\infty\}\|_{L_{\Phi}}},
$$

then

$$
\left\|a^{*}\right\|_{Y^{\prime}} \leq c_{0}\|a\|_{Y^{\prime}} \leq \frac{\left\|\chi_{\{v<\infty\}}\right\|_{Y^{\prime}}}{\left\|\chi_{\{v<\infty\}}\right\|_{L_{\Phi}}},
$$

which implies $a$ is a $\left(\Phi, Y^{\prime}\right)^{*}$-atom. Let $\mu=\frac{2\left\|\chi_{\{v<\infty\}}\right\|_{L_{\Phi}}}{\left\|\chi_{\{v<\infty\}}\right\|_{Y^{\prime}}}$, then

$$
\mathcal{A}_{Y^{\prime}}^{*}(\mu, a, v)=\inf \left\{c>0: P(v<\infty) \Phi\left(\frac{\mu\left\|a^{*}\right\|_{Y^{\prime}}}{c\|\chi\{v<\infty\}\|_{Y^{\prime}}}\right) \leq 1\right\} \leq \mu .
$$


Indeed, this is because

$$
\begin{aligned}
P(v<\infty) \Phi\left(\frac{\mu\left\|a^{*}\right\|_{Y^{\prime}}}{\mu\left\|\chi_{\{v<\infty\}}\right\|_{Y^{\prime}}}\right) & \leq P(v<\infty) \Phi\left(\frac{2}{\mu\left\|\chi_{\{v<\infty\}}\right\|_{Y^{\prime}}}\right) \\
& =P(v<\infty) \Phi\left(\frac{2\left\|\chi_{\{v<\infty\}}\right\|_{Y^{\prime}}}{2\left\|\chi_{\{v<\infty\}}\right\|_{Y^{\prime}}\left\|\chi_{\{v<\infty\}}\right\|_{L_{\Phi}}}\right) \\
& =P(v<\infty) \Phi\left(\Phi^{-1}(1 / P(v<\infty))\right) \\
& =1
\end{aligned}
$$

Since $\beta_{Y^{\prime}}=1-\alpha_{Y}<1$, then by Remark 3.6 (2)

$$
h-h^{v} \in H_{\Phi}
$$

with its quasi-norm

$$
\left\|h-h^{v}\right\|_{H_{\Phi}} \leq c \mu .
$$

By [26], if $\left(\mathcal{F}_{n}\right)_{n \geq 1}$ is regular, $\left(H_{\Phi}\right)^{*}=\left(H_{\Phi}^{s}\right)^{*}=B M O_{\phi, 2}=B M O_{\phi, 1}$. Therefore,

$$
\begin{aligned}
\frac{1}{\phi(P(v<\infty))} \frac{\left\|\left(f-f^{v}\right) \chi_{\{v<\infty\}}\right\|_{Y}}{\left\|\chi_{\{v<\infty\}}\right\|_{Y}} & \leq \frac{1}{\phi(P(v<\infty))} \frac{2\left|\int_{\{v<\infty\}}\left(f-f^{v}\right) h d P\right|}{\left\|\chi_{\{v<\infty\}}\right\|_{Y}} \\
& =\frac{1}{\phi(P(v<\infty))} \frac{2\left|\int_{\{v<\infty\}} f\left(h-h^{v}\right) d P\right|}{\left\|\chi_{\{v<\infty\}}\right\|_{Y}} \\
& \leq \frac{2}{\phi(P(v<\infty))} \frac{\left\|h-h^{v}\right\|_{H_{\Phi}}\|f\|_{B M O_{\phi, 2}}}{\left\|\chi_{\{v<\infty\}}\right\|_{Y}} \\
& \leq c \frac{\left\|\chi_{\{v<\infty\}}\right\|_{L_{\Phi}}}{\phi(P(v<\infty)) P(v<\infty)}\|f\|_{B M O_{\phi, 1}} \\
& =c\|f\|_{B M O_{\phi, 1}},
\end{aligned}
$$

which means

$$
\|f\|_{B M O_{\phi, Y}} \leq c\|f\|_{B M O_{\phi, 1}} .
$$

3.2. John-Nirenberg inequalities with variable exponents. In this subsection we prove $B M O_{\phi, p(\cdot)}=B M O_{\phi, 1}$. We first state an extension of the converse Hölder inequality, which will be used to deal with variable exponent inequalities.

LEMMA 3.8. Let $p(\cdot) \in \mathcal{P}, 0<p_{-} \leq p_{+}<\infty$ and satisfy (1.3). Then for all set $B \in \mathcal{F}$, we have

$$
\mathbb{P}(B)^{1 / p_{-}(B)} \approx \mathbb{P}(B)^{1 / p(x)} \approx \mathbb{P}(B)^{1 / p_{+}(B)} \approx\left\|\chi_{B}\right\|_{p(\cdot)} \quad \forall x \in B .
$$


Proof. Obviously, we have $\mathbb{P}(B)^{1 / p_{-}(B)} \leq \mathbb{P}(B)^{1 / p(x)} \leq \mathbb{P}(B)^{1 / p_{+}(B)}$, for all $x \in B$. Since (1.3), we have

$$
\frac{\mathbb{P}(B)^{1 / p(x)}}{\mathbb{P}(B)^{1 / p_{-}(B)}} \leq \mathbb{P}(B)^{\frac{p_{-}(B)-p(x)}{p_{-}(B) p(x)}} \leq K_{p(\cdot)}^{\frac{1}{p_{-}^{2}(\Omega)}} \triangleq K .
$$

This implies $\mathbb{P}(B)^{1 / p(x)} \leq K \mathbb{P}(B)^{1 / p_{-}(B)}$.

Then it is easy to check that $\mathbb{P}(B)^{1 / p_{-}(B)} \approx \mathbb{P}(B)^{1 / p(x)} \approx \mathbb{P}(B)^{1 / p_{+}(B)}$. And we have

$$
\frac{\chi_{B}(x)}{\mathbb{P}(B)^{1 / p_{-}(B)}} \approx \frac{\chi_{B}(x)}{\mathbb{P}(B)^{1 / p(x)}},
$$

that is

$$
\left(\frac{\chi_{B}(x)}{\mathbb{P}(B)^{1 / p_{-}(B)}}\right)^{p(x)} \geq \frac{\chi_{B}(x)}{\mathbb{P}(B)} \geq\left(\frac{\chi_{B}(x)}{K \mathbb{P}(B)^{1 / p_{-}(B)}}\right)^{p(x)}
$$

So

$$
\int_{\Omega}\left(\frac{\chi_{B}(x)}{\mathbb{P}(B)^{1 / p_{-}(B)}}\right)^{p(x)} d \mathbb{P} \approx \int_{\Omega} \frac{\chi_{B}(x)}{\mathbb{P}(B)} d \mathbb{P}=1 .
$$

Consequently, we get $\left\|\chi_{B}\right\|_{p(\cdot)} \approx \mathbb{P}(B)^{1 / p_{-}(B)}$ and we get the desired result.

COROllary 3.9. Let $p(\cdot) \in \mathcal{P}$ and satisfy (1.3).

(1) Then for all set $B \in \mathcal{F}$, we have

$$
\left\|\chi_{B}\right\|_{1} \approx\left\|\chi_{B}\right\|_{p(\cdot)}\left\|\chi_{B}\right\|_{q(\cdot)},
$$

where

$$
1=\frac{1}{p(x)}+\frac{1}{q(x)} .
$$

(2) Let $q(\cdot) \in \mathcal{P}$ and satisfies (1.3). Then for all set $B \in \mathcal{F}$, we have

$$
\left\|\chi_{B}\right\|_{r(\cdot)} \approx\left\|\chi_{B}\right\|_{p(\cdot)}\left\|\chi_{B}\right\|_{q(\cdot)},
$$

where

$$
\frac{1}{r(x)}=\frac{1}{p(x)}+\frac{1}{q(x)} .
$$

PRoOF. It follows from Lemma 3.8 that

$$
\left\|\chi_{B}\right\|_{r(\cdot)} \approx \mathbb{P}(B)^{\frac{1}{r(x)}}=\mathbb{P}(B)^{\frac{1}{p(x)}+\frac{1}{q(x)}} \approx\left\|\chi_{B}\right\|_{p(\cdot)}\left\|\chi_{B}\right\|_{q(\cdot)}, \quad \forall x \in B .
$$

THEOREM 3.10. Let $p(\cdot) \in \mathcal{P}$ and satisfy the condition (1.3). If $\left(\mathcal{F}_{n}\right)_{n \geq 1}$ is regular, then

$$
B M O_{\phi, p(\cdot)}=B M O_{\phi, 1}
$$


where $\phi(r)=\frac{1}{r \Phi^{-1}(1 / r)}$ and $\Phi \in \mathfrak{S}_{\ell}$.

PROOF. First suppose that $f \in B M O_{\phi, p(\cdot)}$. Then for $v \in \mathcal{T}$, by Lemma 2.7 and Corollary 3.9, we have

$$
\begin{aligned}
& \frac{1}{\phi(P(v<\infty))} \frac{\left\|\left(f-f^{v}\right) \chi_{\{v<\infty\}}\right\|_{1}}{P(v<\infty)} \\
\lesssim & \frac{1}{\phi(P(v<\infty))} \frac{\left\|\left(f-f^{v}\right) \chi_{\{v<\infty\}}\right\|_{p(\cdot)}\left\|\chi_{\{v<\infty\}}\right\|_{p^{\prime}(\cdot)}}{P(v<\infty)} \\
= & \frac{1}{\phi(P(v<\infty))} \frac{\left\|\left(f-f^{v}\right) \chi_{\{v<\infty\}}\right\|_{p(\cdot)}}{\left\|\chi_{\{v<\infty\}}\right\|_{p(\cdot)}} \cdot \frac{\left\|\chi_{\{v<\infty\}}\right\|_{p(\cdot)}\left\|\chi_{\{v<\infty\}}\right\|_{p^{\prime}(\cdot)}}{\left\|\chi_{\{v<\infty\}}\right\|_{1}} \\
\lesssim & \|f\|_{B M O_{\phi, p(\cdot)}}
\end{aligned}
$$

This means $\|f\|_{B M O_{\phi, 1}} \lesssim\|f\|_{B M O_{\phi, p(\cdot)}}$.

On the other hand, assume that $f \in B M O_{\phi, 1}$. Then for $v \in \mathcal{T}$, by Lemma 2.7, we have

$$
\begin{aligned}
\left\|\left(f-f^{v}\right) \chi_{\{v<\infty\}}\right\|_{p(\cdot)} & \lesssim\left\|\left(f-f^{v}\right) \chi_{\{v<\infty\}}\right\|_{p_{+}}\left\|\chi_{\{v<\infty\}}\right\|_{\frac{p_{+} p(\cdot)}{p_{+}-p(\cdot)}} \\
& =\frac{\left\|\left(f-f^{v}\right) \chi_{\{v<\infty\}}\right\|_{p_{+}}}{\left\|\chi_{\{v<\infty\}}\right\|_{p_{+}}}\left\|\chi_{\{v<\infty\}}\right\|_{\frac{p_{+} p(\cdot)}{p_{+}-p(\cdot)}}\left\|\chi_{\{v<\infty\}}\right\|_{p_{+}} .
\end{aligned}
$$

Hence, we can get

$$
\frac{1}{\phi(P(v<\infty))}\left\|\left(f-f^{v}\right) \chi_{\{v<\infty\}}\right\|_{p(\cdot)} \lesssim\|f\|_{B M O_{\phi, p_{+}}}\left\|\chi_{\{v<\infty\}}\right\|_{\frac{p+p(\cdot)}{p_{+}-p(\cdot)}}\left\|\chi_{\{v<\infty\}}\right\|_{p_{+}} .
$$

Then from Theorem 2.9 in [26], we have

$$
\frac{1}{\phi(P(v<\infty))}\left\|\left(f-f^{v}\right) \chi_{\{v<\infty\}}\right\|_{p(\cdot)} \lesssim\|f\|_{B M O_{\phi, 1}}\left\|\chi_{\{v<\infty\}}\right\|_{\frac{p+p(\cdot)}{p_{+}-p(\cdot)}}\left\|\chi_{\{v<\infty\}}\right\|_{p_{+}} .
$$

Henceforth, by Corollary 3.9,

$$
\begin{aligned}
\frac{1}{\phi(P(v<\infty))} \frac{\left\|\left(f-f^{v}\right) \chi_{\{v<\infty\}}\right\|_{p(\cdot)}}{\left\|\chi_{v<\infty}\right\|_{p(\cdot)}} & \lesssim\|f\|_{B M O_{\phi, 1}} \frac{\left\|\chi_{\{v<\infty\}}\right\|_{\frac{p_{+} p(\cdot)}{p_{+}-p(\cdot)}}\left\|\chi_{\{v<\infty\}}\right\|_{p_{+}}}{\left\|\chi_{\nu<\infty}\right\|_{p(\cdot)}} \\
& \lesssim\|f\|_{B M O_{\phi, 1}},
\end{aligned}
$$

which means

$$
\|f\|_{B M O_{\phi, p(\cdot)}} \leq c\|f\|_{B M O_{\phi, 1}} .
$$

According to Theorems 3.7 and 3.10, we get Theorem 3.1.

REMARK 3.11. 1) If $p(\cdot) \equiv 1$ and $\phi(r) \equiv 1$ for $r \in(0, \infty)$, then Theorem 3.1 reduces to the main result recently obtained in [32].

2) If $p(\cdot) \equiv 1$ and $Y=L_{q}$, then Theorem 3.1 reduces to (1.2) in the first section. 
ACKNOWLEDGEMENTS. The authors would like to thank the referee and the editorial committee for their careful reading and useful comments.

\section{References}

[ 1 ] A. AlmeIdA and P. HÄstö, Besov spaces with variable smoothness and integrability, J. Funct. Anal. 258 (2010), 1628-1655.

[ 2 ] C. Bennett and R. Sharpley, Interpolation of operators, Academic Press, New York, 1988.

[ 3 ] D. Cruz-Uribe and A. Fiorenza, Variable Lebesgue Spaces: Foundations and Harmonic Analysis, Birkhäuser, 2013.

[ 4 ] G. CUERVA, Weighted norm inequalities and related topics, North-Holland, Amsterdam, 1985.

[ 5 ] D. CRUZ-URIBE, SFO and L. DANIEl WANG, Variable Hardy spaces, to appear in Indiana University Mathematics Journal, arXiv:1211.6505v1, 2014.

[ 6] L. Diening and P. Harjulehto, Lebesgue and Sololev spaces with variable exponent, Springer, 2011.

[ 7 ] L. Diening, Maximal functions on generalized $L^{p(\cdot)}$ spaces, Math. Inequal. Appl. 7(2) (2004), 245-253.

[ 8 ] L. Diening, P. HÄstö and R. Roudenko, Function spaces of variable smoothness and integrability, J. Funct. Anal. 256 (2009), 1731-1768.

[ 9 ] D. BoYD, Indices of function spaces and their relationship to interpolation, Canad. J. Math. 21 (1969), 1245-1254.

[10] X. FAn and D. ZhaO, On the spaces $L^{p(x)}(\Omega)$ and $W^{m, p(x)}$, J. Math. Anal. Appl. 263 (2001), 424-446.

[11] A. M. GARsia, Martingale inequalities: Seminar notes on recent progress, Math. Lecture Notes Series, New York: Benjamin Inc., 1973.

[12] C. Herz, Bounded mean oscillation and regulated martingales, Trans. Amer. Math. Soc. 193 (1974), 199-215.

[13] P. Harjulehto, P. Hästö and M. Pere, Variable exponent Lebesgue spaces on metric spaces: The HardyLittlewood maximal operator, Real Anal. Exchange 30(1) (2004), 87-104.

[14] R. L. Long, Martingale Spaces and Inequalities, Peking University Press, Beijing, 1993.

[15] Y. JiAO, Carleson measures and vector-valued BMO martingales, Probab. Theory Relat. Fields 145 (2009), 421-434.

[16] Y. JIAO, L. WU and M. POPA, Operator-valued martingale transforms in rearrangement invariant spaces and applications, Sci. China Math. 56(4) (2013), 831-844.

[17] Y. Jiao, D. Zhou, Z. HaO and W. Chen, Martingale Hardy spaces with variable exponents, arXiv:1404.2395v2, 2014.

[18] F. John and L. Nirenberg, On the functions of bounded mean oscillation, Comm. Pure Appl. Math. 14 (1961), 415-426.

[19] W.B. Johnson and G. Schechtman, Martingale inequalities in rearrangement invariant function spaces, Israel J. Math. 64(3) (1998), 267-275.

[20] M. IZUKI and Y. SAWANO, Variable Lebesgue norm estimates for BMO functions, Czechoslovak Math. J. 62 (137) (2012), 717-727.

[21] M. IZUKI, Y. SAWANO and Y. TsUTSUI, Variable Lebesgue norm estimates for BMO functions II preprint, 2013.

[22] K. P. Ho, John-Nirenberg inequalities on Lebesgue spaces with variable exponents, Taiwanese J. Math. 18(4) (2014), 1107-1118.

[23] M. KIKUCHI, New martingale inequalities in rearrangement function spaces, Proc. Edinb. Math. Soc. 47(2) (2004), 633-657.

[24] S. Krein, Y. Petunin and E. Semenov, Interpolation of linear operators, Amer. Math. Soc., Providence, 1982.

[25] O. KovÀC̆IK and J. RÁKosNíK, On spaces $L^{p(x)}$ and $W^{1, p(x)}$, Czechoslovak Math. J. 41(116) (1991), 592618. 
[26] T. Miyamoto, E. Nakai and G. Sadasue, Martingale Orlicz-Hardy spaces, Math. Nachr. 285 (2012), 670-686.

[27] E. NAKai and G. Sadasue, Martingale Morrey-Campanato spaces and fractional integrals, J. Funct. Spaces Appl. (2012) Article ID673929, 29 pp.

[28] N. I. YA, Martingale inequalities in rearrangement invariant function spaces, In Function spaces, TeubnerTexte Math., Teubnet, Stuttgart, 120 (1991), 120-127.

[29] E. NAKAI and Y. SAWANO, Hardy spaces with variable exponents and generalized Campanato spaces, J. Funct. Anal. 262 (2012), 3665-3748.

[30] W. ORLICZ, Über konjugierte Expoentenfolgen, Studia Math. 3 (1931), 200-211.

[31] F. WEISZ, Martingale Hardy spaces and their applications in Fourier analysis, Lecture Notes in Mathematics, 1568, Springer-Verlag, Berlin, 1994.

[32] R. YI, L. WU and Y. JIAO, New John-Nirenberg inequalities for martingales, Statist. Probab. Lett. 86 (2014), $68-73$.

Present Addresses:

LIAN WU

SCHOOL OF MATHEMATics AND STATISTICS,

Central South University,

CHANGSHA 410075, CHINA.

ZHIWEI HAO

School of Mathematics and Statistics,

CEnTral South UNIVERSity,

Changsha 410075, China.

YONG JIAO

SChool of Mathematics and Statistics,

Central South University,

CHANGSHA 410075, CHINA. 